\title{
OPEN Factors influencing self-selected walking speed in fibrotic interstitial lung disease
}

\author{
Gabriela Fischer ${ }^{1,2}$, Francisco B. de Queiroz ${ }^{2}$, Danilo C. Berton ${ }^{2}$, Pedro Schons ${ }^{2,3,4}$, \\ Henrique B. Oliveira ${ }^{3,4}$, Marcelo Coertjens ${ }^{2,3,5}$, Mathieu Gruet $^{6}$ \& \\ Leonardo A. Peyré-Tartaruga $a^{2,3,4 \bowtie}$
}

This study aimed to investigate the walking economy and possible factors influencing self-selected walking speed (SSWS) in patients with fibrotic interstitial lung disease (ILD) compared to controls. In this study, 10 patients with ILD (mean age: $63.8 \pm 9.2$ years, forced expiratory volume in the first second: $56 \pm 7 \%$ of predicted) and 10 healthy controls underwent resting pulmonary function tests, cardiopulmonary exercise, and submaximal treadmill walking tests at different speeds. The walking economy was assessed by calculating the cost-of-transport (CoT). Dynamic stability was assessed by stride-to-stride fluctuations using video recordings. Patients with ILD showed reduced peak oxygen uptake with a tachypneic breathing pattern and significant oxygen desaturation during exercise. The CoT did not differ between the groups $(p=0.680)$, but dyspnea and $\mathrm{SpO}_{2}$ were higher and lower, respectively, in patients with ILD at the same relative speeds. SSWS was reduced in ILD patients $\left(2.6 \pm 0.9\right.$ vs. $\left.4.2 \pm 0.4 \mathrm{~km} \mathrm{~h}^{-1} p=0.001\right)$ and did not correspond to the energetically optimal walking speed. Dynamic stability was significantly lower in patients with ILD than in healthy controls, mainly at lower speeds. Patients with ILD presented a similar cost of transport compared to healthy controls; however, they chose lower SSWS despite higher walking energy expenditure. Although walking stability and dyspnea were negatively affected, these factors were not associated with the slower walking speed chosen by individuals with ILD.

Fibrotic interstitial lung disease (ILD) is a pathologically heterogeneous group of diseases ${ }^{1-3}$ characterized by impaired pulmonary diffusive potential and ventilatory mechanics ${ }^{4,5}$. In patients with fibrotic ILD, the progression of hypoxemia and dyspnea are associated with disability, reduced health-related quality of life, and reduced survival ${ }^{6}$.

As the disease progresses, breathlessness can impair the functional status of these patients, limiting daily activities $^{6}$. In fact, functional capacity, assessed by field walking tests, is impaired and inversely related to the magnitude of dyspnea ${ }^{7,8}$. Interestingly, patients with reduced usual walking speed have significantly worse exercise performance and health status, despite similar lung function and fibrotic tomographic extension ${ }^{7}$. Although field walking tests are easy to perform and widely used in clinical practice ${ }^{9}$, they are unable to further our understanding of the mechanisms underlying reduced walking performance. Exercise test protocols assessing the energy economy profile and dynamic stability during walking speeds in daily life may provide more clarity ${ }^{10}$.

The cost of transport (CoT) is a movement-economy indicator that describes the mass-specific energy consumption required to travel a certain distance ${ }^{11}$. Individuals are more economical when they spend less energy to cover a given distance. Several studies ${ }^{11-13}$ have shown that the CoT depends on walking speed and that there is an optimal walking speed (OWS) for healthy young individuals $\left(\approx 4 \mathrm{~km} \mathrm{~h}^{-1}\right)$, where the energy expenditure is minimal and corresponds to the self-selected walking speed (SSWS). However, in certain conditions, the CoT is not minimized at SSWS, such as on rough terrain ${ }^{14}$ and while walking downhill ${ }^{15}$, where individuals prefer a more stable and costly gait pattern. In the elderly, SSWS matches the OWS, despite a higher CoT than that in the

\footnotetext{
${ }^{1}$ Biomechanics Laboratory, Federal University of Santa Catarina, Florianópolis, SC, Brazil. 2Programa de Pós-Graduação Em Ciências Pneumológicas, Hospital de Clínicas de Porto Alegre/Universidade Federal Do Rio Grande Do Sul, Porto Alegre, RS, Brazil. ${ }^{3}$ Exercise Research Laboratory, Universidade Federal Do Rio Grande Do Sul, Rua Felizardo, 750, Porto Alegre, RS 90690-200, Brazil. ${ }^{4}$ Programa de Pós-Graduação Em Ciências Do Movimento Humano, Universidade Federal Do Rio Grande Do Sul, Porto Alegre, RS, Brazil. ${ }^{5}$ Programa de Pós-Graduação Em Ciências Biomédicas, Universidade Federal Do Delta Do Parnaíba, Parnaíba, PI, Brazil. '́Laboratoire IAPS, Université de Toulon, 83041 Toulon, France. ${ }^{\circledR}$ email: leonardo.tartaruga@ufrgs.br
} 
young individuals ${ }^{16}$. The lower economic walking performance in the elderly is supposed to be due an unstable walking pattern and sarcopenia/muscle weakness associated with aging ${ }^{17,18}$.

Studies on skeletal muscle dysfunction ${ }^{19-21}$ and impaired functional capacity ${ }^{22,23}$ associated with ILD suggest an even worse scenario. It is increasingly recognized that aging and related extrapulmonary manifestations, such as cardiovascular diseases, contribute to morbidity in ILD ${ }^{8}$. In patients with cardiopulmonary disorders, the SSWS is lower than the OWS and is lower than that selected by age- and sex-matched healthy controls. Chronic heart failure patients choose a SSWS with a higher CoT but with lower ventilatory $\operatorname{cost}^{24}$. In patients with chronic obstructive pulmonary disease (COPD), the reason for choosing a less economic SSWS seems to be mediated by the balance between lower dyspnea perception and higher gait stability ${ }^{10}$. It is unknown whether similar locomotor reasons exist among patients with ILD. Despite of disease-specific pulmonary differences including respiratory mechanics and the activity of various respiratory muscles, the dyspnea and inspiratory neural drive relationship during exercise are similar between ILD and COPD patients ${ }^{25,26}$.

The aim of this study was to investigate the walking economy and the possible factors influencing SSWS adopted by patients with ILD in comparison to healthy controls. We hypothesized that patients would select their usual walking speed (i.e. SSWS) to achieve a balance between their higher dyspnea and lower gait stability regardless of the most economic walking speed (i.e. OWS).

\section{Methods}

Subjects and ethics statement. This study included 10 patients with established fibrotic ILD (three with idiopathic pulmonary fibrosis, four with fibrotic non-specific interstitial pneumonia, and three with chronic hypersensitivity pneumonia) and 10 healthy controls matched for age and sex. Respirologists diagnosed ILD based on the patients' clinical characteristics, pulmonary function, high-resolution chest tomography results, and in some cases, lung biopsy results. Patients with an unstable clinical condition who presented with comorbidities such as severe cardiovascular and neuromuscular diseases or musculoskeletal and/or joint injuries that impaired treadmill walking were excluded from the study. Patients on long-term oxygen therapy or those who were unable to complete the tests were also excluded. All participants read and signed an informed consent form. The study protocol was approved by the Ethics Committee of the Hospital de Clínicas de Porto Alegre (150,550).

Study design. A cross-sectional study was conducted on two different days. On the first day, resting pulmonary function tests and incremental cardiopulmonary exercise tests were performed. On the second day, treadmill walking tests were performed at different speeds to determine CoT and dynamic stability.

Pulmonary function test. Pulmonary function tests were performed using a computerized system (Jaeger v. 4.31, Jaeger, Wuerzburg, Germany). Forced vital capacity (FVC), forced expiratory volume in the first second $\left(\mathrm{FEV}_{1}\right), \mathrm{FEV}_{1} \mathrm{FVC}^{-1}$ ratio, diffusing capacity of the lung for carbon monoxide $\left(\mathrm{DL}_{\mathrm{CO}}\right.$ ), and total lung capacity (TLC) were recorded and expressed as the percentage of predicted values. All tests were performed according to international standards.

Cardiopulmonary exercise testing. Cardiopulmonary exercise tests were performed on an ergometric treadmill (General Electric T2100, Fairfield, CA, USA), using a ramp protocol. Participants completed walking familiarization on the treadmill at low speeds and received instructions on the test. They were then fitted with a mask for breath-by-breath gas analyses (Vmax Encore PFT system, CA, USA), electrodes for ECG (ECG 12-lead CASE system-Cardiosoft, GE Healthcare, Wauwatosa, USA) were placed, and oxygen saturation $\left(\mathrm{SpO}_{2}\right)$ by pulse oximetry was continuously monitored (Nellcor Puritan Bennett-NPB-195, Pleasanton, CA, USA). Patients with ILD and the controls performed different protocols according to their physical fitness to obtain a test duration between 8 and $12 \mathrm{~min}^{27}$. The protocol for patients with ILD consisted of a rest period followed by a warm-up of $3 \mathrm{~min}$ at $1.5 \mathrm{~km} \mathrm{~h}^{-1}$. The incremental phase began with $2 \mathrm{~km} \mathrm{~h}^{-1}$ and $1 \%$ inclination with increments of $0.5 \mathrm{~km} \mathrm{~h}^{-1}$ per minute until the fourth minute. After that, the speed remained constant, and the slope of the treadmill was increased by $2 \%$ every minute until volitional exhaustion. In the control group protocol, the warmup speed was $3 \mathrm{~km} \mathrm{~h}^{-1}$, and the incremental phase began with $4 \mathrm{~km} \mathrm{~h}^{-1}$ and $1 \%$ inclination with increments of $0.5 \mathrm{~km} \mathrm{~h}^{-1}$ per minute until the fourth minute. The remaining part of the exercise protocol was similar to that followed by patients with ILD. Dyspnea and leg effort were rated at the end of each stage using the Borg CR-10 scale $^{28}$, and maximal effort was confirmed according to standard physiological and psychological criteria ${ }^{29}$. Peak oxygen uptake $\left(\dot{\mathrm{VO}}_{2 \text { peak }}\right)$ was defined as a $20 \mathrm{~s}$ average around the highest value during the test ${ }^{27}$.

Treadmill walking tests. The participants completed 5-10 min of familiarization on the ground and treadmill. The SSWS was first determined as the participants walked across a $15 \mathrm{~m}$ hallway three times at a comfortable pace. Times were registered using a stopwatch for a length of $10 \mathrm{~m}$ to avoid acceleration and deceleration phases. The reliability was tested extensively using two or three repetitions ${ }^{30}$. The SSWS was calculated by dividing the distance by the time required to cover it. The SSWS tested on the ground was considered as the average value. The SSWS was then tested and determined on a treadmill where a trained evaluator provided instructions for the participants. The treadmill speed indicator panel was covered, so that the participants did not know the speed at which they were walking. Thus, the researchers instructed the participants to walk at their most comfortable speed. The treadmill speed was gradually decreased or increased by $0.5 \mathrm{~km} \mathrm{~h}^{-1}$ several times, until the participants were sure that the speed was their most comfortable walking speed. Only after this confirmation was the treadmill panel uncovered and the SSWS registered ${ }^{31}$. The SSWS was adjusted on the treadmill at a comfortable pace and then four other speeds were defined: two speeds above $(+20 \%$ and $+40 \%)$ and two speeds below $(-20 \%$ and $-40 \%)$ the SSWS. Additionally, all participants walked through an iso-speed of $3.2 \mathrm{~km} \mathrm{~h}^{-1}$. 
Participants walked at six different speeds for $5 \mathrm{~min}$ in a randomized order with the intervals between the trials large enough to return to a heart rate of $<100 \mathrm{bpm}$. Cardiorespiratory parameters (Vmax ${ }^{\oplus}$ Encore) and $\mathrm{SpO}_{2}$ were recorded throughout the data collection period. Dyspnea and fatigue of the lower limbs (Borg CR-10) ${ }^{26}$ were recorded at the end of the $5 \mathrm{~min}$ trials. The spatiotemporal variables were continuously recorded using a 2D kinematics technique. A video camera $(120 \mathrm{~Hz}$, CASIO Exilim FH25, Tokyo, Japan) was positioned perpendicular to the side of the treadmill. The step phase was defined using video recording cameras and three reflective markers on the fifth metatarsal, calcaneus, and lateral malleolus.

Data processing. Cost of transport, optimal speed, and locomotor rehabilitation index. The average of $\dot{\mathrm{V}} \mathrm{O}_{2}$ $\left(\mathrm{ml} \mathrm{kg}^{-1} \mathrm{~min}^{-1}\right)$ obtained from the last minute of each walking speed was used to calculate $\mathrm{CoT}^{12}$. This value was subtracted from the mean standing $\dot{\mathrm{V}} \mathrm{O}_{2}$ and transformed into Joules $(\mathrm{J})$ using the equation: metabolic power $=$ $\dot{\mathrm{VO}}_{2}(4.94 \mathrm{RER}+16.04) / 60\left(\mathrm{~J} \mathrm{~kg}^{-1} \mathrm{~s}^{-1}\right)$, where RER was the respiratory exchange ratio ${ }^{12}$. Finally, $\mathrm{CoT}\left(\mathrm{J} \mathrm{kg}^{-1} \mathrm{~m}^{-1}\right)$ was calculated as the ratio of metabolic energy $\left(\mathrm{J} \mathrm{kg}^{-1} \mathrm{~s}^{-1}\right)$ and walking speed $\left(\mathrm{m} \mathrm{s}^{-1}\right)$. Ventilation minutes $\left(\dot{\mathrm{V}}_{\mathrm{E}}\right)$ were divided by the $\dot{\mathrm{V} C O}$ (ventilatory equivalent for carbon dioxide) to determine the ventilatory efficiency ${ }^{24}$.

The OWS (i.e., the walking speed at the minimum CoT) was determined using a second-degree polynomial function: ${ }^{32}$

$$
\text { OWS }=-\mathrm{b} \times 2 \mathrm{a}^{-1}
$$

where $\mathrm{a}$ and $\mathrm{b}$ are empirical constants based on CoT as a function of speed from the experimental data obtained from the control and ILD groups, respectively.

The locomotor rehabilitation index represents the similarity of the SSWS to the biomechanical OWS. The biomechanical OWS is calculated as the square root of the product of the Froude number at the OWS (i.e., 0.25), gravitational acceleration $\left(9.81 \mathrm{~m} \mathrm{~s}^{-2}\right)$, and leg length. The locomotor rehabilitation index is the ratio of the SSWS to biomechanical OWS, multiplied by $100 \%{ }^{33}$.

Dynamic stability. The spatiotemporal variables of contact time, swing time, stride frequency, and stride length were obtained by analyzing 10 cycles of the last-minute walking for each speed using the Kinovea program (0.8.15, Montceau-les-Mines, France). The analysis consisted of determining the frames in which the foot touched the ground and when the foot ceased to have contact with the ground (i.e., unloading), as determined by a trained researcher ${ }^{34}$.

Dynamic stability was calculated using the coefficient of variation $(\mathrm{CoV})$. The stride-to-stride fluctuations were the result of the division between the standard deviation and the mean of the contact time, swing time, stride frequency, and stride length, from at least 10 strides, using a customized algorithm constructed using the LabVIEW software (version 8.5, National Instruments, Austin, TX, USA). The table contains sheets including individual data for all variables in Supplementary Material 01.

Statistical analyses. Descriptive statistics were used, and data are presented as means and standard deviations. The normal distribution of the data was verified using the Shapiro-Wilk test. Student's $t$ tests for independent samples were used to compare pulmonary function and cardiopulmonary exercise test variables between the patients and controls. A two-way repeated-measures ANOVA was used to compare CoT, Borg dyspnea, $\dot{\mathrm{V}}$ $\mathrm{E} / \dot{\mathrm{V} C O}{ }_{2}$, and dynamic stability. The two factors were the group (patient and control) and speed $(-40 \%,-20 \%$, SSWS, $+20 \%,+40 \%$, and $3.2 \mathrm{~km} \mathrm{~h}^{-1}$ ). If a significant interaction between the group and speed was found, post hoc $t$-tests for independent samples and the Bonferroni test were performed. Statistical analyses were performed using the SPSS version 20. The level of significance was set at $p \leq 0.05$.

\section{Results}

Age, body mass, height, and body mass index did not differ between the groups $(p>0.05)$. The patients showed an average reduction in TLC and $\mathrm{DL}_{\mathrm{CO}}$ (Table 1). Table 2 shows the cardiorespiratory variables obtained from the cardiopulmonary exercise test. Patients with ILD presented a reduced $\dot{\mathrm{V}} \mathrm{O}_{2 \text { peak }}$ compared to that of controls $(p=0.001)$, with a tachypneic breathing pattern and significant oxygen desaturation during exercise.

ILD patients adopted a significantly lower SSWS compared to controls $\left(2.6 \pm 0.9 \mathrm{vs} 4.2 \pm 0.4 \mathrm{~km} \mathrm{~h}^{-1}\right.$, respectively; $p=0.001$ ). OWS and the associated CoT were $3.96 \mathrm{~km} \mathrm{~h}^{-1}$ and $2.91 \mathrm{~J} \mathrm{~kg}^{-1} \mathrm{~m}^{-1}$, respectively, for ILD patients and $5.06 \mathrm{~km} \mathrm{~h}^{-1}$ and $2.72 \mathrm{~J} \mathrm{~kg}^{-1} \mathrm{~m}^{-1}$, respectively for controls, while the resulting locomotor rehabilitation index was $53 \pm 18 \%$ for ILD patients and $84 \pm 10 \%$ for controls.

Figure 1 shows CoT as a function of the absolute speeds, while Fig. 2 shows CoT, dyspnea, and $\mathrm{SpO}_{2}$ as a function of the relative speeds: $+40 \%,+20 \%$, SSWS, $20 \%$, and $-40 \%$. CoT was not significantly different between the groups $(p=0.680)$ or speeds $(p=0.139)$. No group $\times$ speed interaction was observed for CoT $(p=0.051)$. Dyspnea was significantly higher in patients with ILD than in the controls $(p=0.005)$ while $\mathrm{SpO}_{2}$ was significantly lower in ILD patients $(89.9 \pm 2.2 \%$ vs. $96 \pm 0.8 \%$, respectively; $p=0.037)$. No differences in dyspnea or $\mathrm{SpO}_{2}$ were observed at different speeds between the groups $(p>0.05)$.

CoT values did not differ between the groups at iso-speed (i.e., $3.2 \mathrm{~km} \mathrm{~h}^{-1}$ ), (patients with ILD $3.18 \pm 0.9 \mathrm{~J}$ $\mathrm{kg}^{-1} \mathrm{~m}^{-1}$ vs. controls $\left.3.48 \pm 1.2 \mathrm{~J} \mathrm{~kg}^{-1} \mathrm{~m}^{-1} ; p=0.555\right)$. However, significant between-group differences were found for dyspnea (patients with ILD $4.3 \pm 3$ vs. controls $0.2 \pm 0.6 ; p=0.002$ ) and $\mathrm{SpO}_{2}$ (patients $88 \pm 10 \%$ vs. controls $97 \pm 1.8 \% ; p=0.022$ ) at $3.2 \mathrm{~km} \mathrm{HP} \mathrm{Inc.}^{-1}$. Ventilatory efficiency was influenced by speed (main effect, $p=0.008$ ) and group (main effect, $p<0.001$ ). Post hoc comparisons showed a reduction in ventilatory efficiency between $-40 \%$ and $+40 \%$ in both groups $(p=0.013)$. Furthermore, no significant interaction was observed (main effect 


\begin{tabular}{|c|c|c|c|}
\hline Variables & ILD $(n=10)$ & Control $(n=10)$ & $p$ \\
\hline Age (years) & $63.8 \pm 9.2$ & $62.2 \pm 8.2$ & 0.685 \\
\hline \multicolumn{4}{|l|}{ Gender (n) } \\
\hline Male & 2 & 2 & \\
\hline Female & 8 & 8 & \\
\hline Body Mass (kg) & $65.2 \pm 10.6$ & $64.9 \pm 12.2$ & 0.248 \\
\hline Height $(\mathrm{cm})$ & $156 \pm 9$ & $162 \pm 8$ & 0.067 \\
\hline BMI $\left(\mathrm{kg} \mathrm{m}^{-2}\right)$ & $27.1 \pm 4.4$ & $24.6 \pm 4.4$ & 0.212 \\
\hline \multicolumn{4}{|l|}{ Lung Function } \\
\hline $\mathrm{FEV}_{1} \cdot \mathrm{L}$ (\%pred) & $1.40 \pm 0.43(56 \pm 7)$ & $2.49 \pm 0.46(92 \pm 16)$ & 0.001 \\
\hline FVC. L (\%pred) & $1.62 \pm 0.55(54 \pm 9)$ & $3.13 \pm 0.44(96 \pm 13)$ & 0.001 \\
\hline $\mathrm{FEV}_{1} / \mathrm{FVC}$ (\%pred) & $0.87 \pm 0.06(116 \pm 16)$ & $0.79 \pm 0.09(107 \pm 21)$ & 0.031 \\
\hline TLC. L (\%pred) & $3.29 \pm 0.72(74 \pm 10)$ & - & - \\
\hline $\mathrm{DL}_{\mathrm{CO} \cdot} \cdot \mathrm{mmol} / \mathrm{min} / \mathrm{kPa}(\%$ pred $)$ & $2.64 \pm 0.58(38 \pm 8)$ & - & - \\
\hline $\mathrm{SpO}_{2} \%$ & $96.8 \pm 2.4$ & $98.5 \pm 1.1$ & 0.117 \\
\hline
\end{tabular}

Table 1. Participants' characteristics (Mean \pm SD). n: number of participants; SD: standard deviation; BMI: body mass index; FVC: forced vital capacity; $\mathrm{FEV}_{1}$ : forced expiratory volume in one second; TLC = total lung capacity; $\mathrm{DL}_{\mathrm{CO}}$ : diffusing capacity of the lung for carbon monoxide; $\mathrm{SpO}_{2}$ : oxyhemoglobin saturation by pulse oximetry.

\begin{tabular}{|c|c|c|c|}
\hline Variables & Patients $(n=10)$ & Controls $(n=10)$ & $p$ \\
\hline$\dot{\mathrm{V}} \mathrm{O}_{2} \mathrm{~L} \min ^{-1}$ (\%pred) & $0.83 \pm 0.47(32 \pm 7)$ & $1.94 \pm 0.36(74 \pm 10)$ & 0.001 \\
\hline$\dot{\mathrm{V}} \mathrm{O}_{2} \mathrm{~mL} \mathrm{~kg}^{-1} \mathrm{~min}^{-1}$ & $12.8 \pm 6.90$ & $28.8 \pm 3.25$ & 0.001 \\
\hline$\dot{\mathrm{V} C O}{ }_{2} \mathrm{~L} \mathrm{~min} \min ^{-1}$ & $0.89 \pm 0.52$ & $2.02 \pm 0.56$ & 0.001 \\
\hline RER & $1.21 \pm 0.21$ & $1.10 \pm 0.08$ & 0.622 \\
\hline$\dot{\mathrm{V}} \mathrm{L} \min ^{-1}$ & $34 \pm 17$ & $61 \pm 18$ & 0.006 \\
\hline$\dot{\mathrm{VE}} / \mathrm{MVV}$ & $0.69 \pm 0.31$ & $0.67 \pm 0.19$ & 0.836 \\
\hline$f$ breaths $\min ^{-1}$ & $48 \pm 11$ & $36 \pm 5$ & 0.003 \\
\hline $\mathrm{V}_{\mathrm{T}}$ & $0.74 \pm 0.40$ & $1.71 \pm 0.35$ & 0.001 \\
\hline HR bpm & $156 \pm 9$ & $158 \pm 8$ & 0.957 \\
\hline $\mathrm{SpO}_{2}$ final $\%$ & $82 \pm 11$ & $95 \pm 1$ & 0.002 \\
\hline Delta $\mathrm{SPO}_{2} \%$ & $-15 \pm 10$ & $-3 \pm 1$ & 0.006 \\
\hline Borg Dyspnea (range) & $3-10$ & $0-9$ & \\
\hline Borg Leg Effort (range) & $0-9$ & $0-10$ & \\
\hline
\end{tabular}

Table 2. Measurements during incremental cardiopulmonary exercise testing (Mean $\pm \mathrm{SD}) . \dot{\mathrm{V}} \mathrm{O}_{2}$ : oxygen consumption; $\mathrm{V}_{\mathrm{CO}_{2}}$ : carbon dioxide output; RER: Respiratory Exchange Ratio; $\dot{\mathrm{VE}}$ : minute ventilation; MVV: maximum voluntary ventilation; $f$ : breathing frequency; $\mathrm{V}_{\mathrm{T}}$ : tidal volume; HR: heart rate; $\mathrm{SpO}_{2}$ : oxyhemoglobin saturation by pulse oximetry.

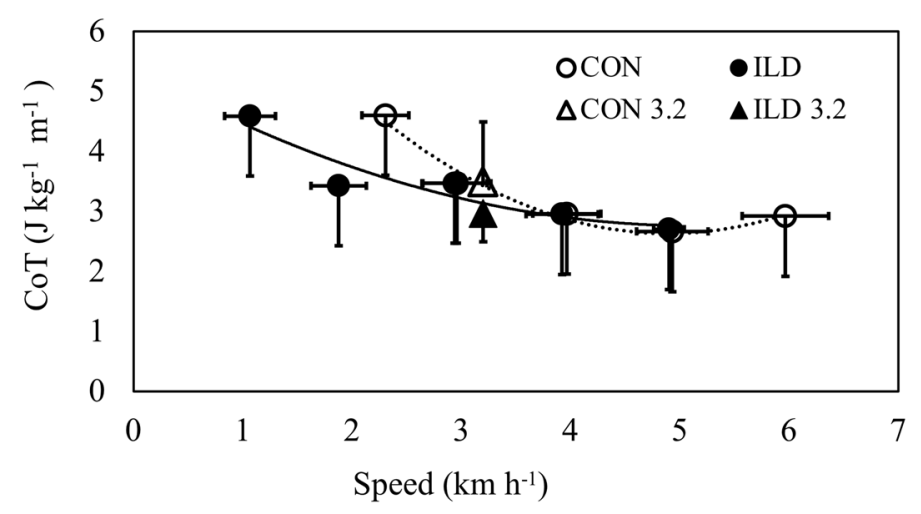

Figure 1. Cost of Transport as a function of absolute speed in patients with interstitial lung disease (ILD, filled circles) and controls (CON, empty circles). 

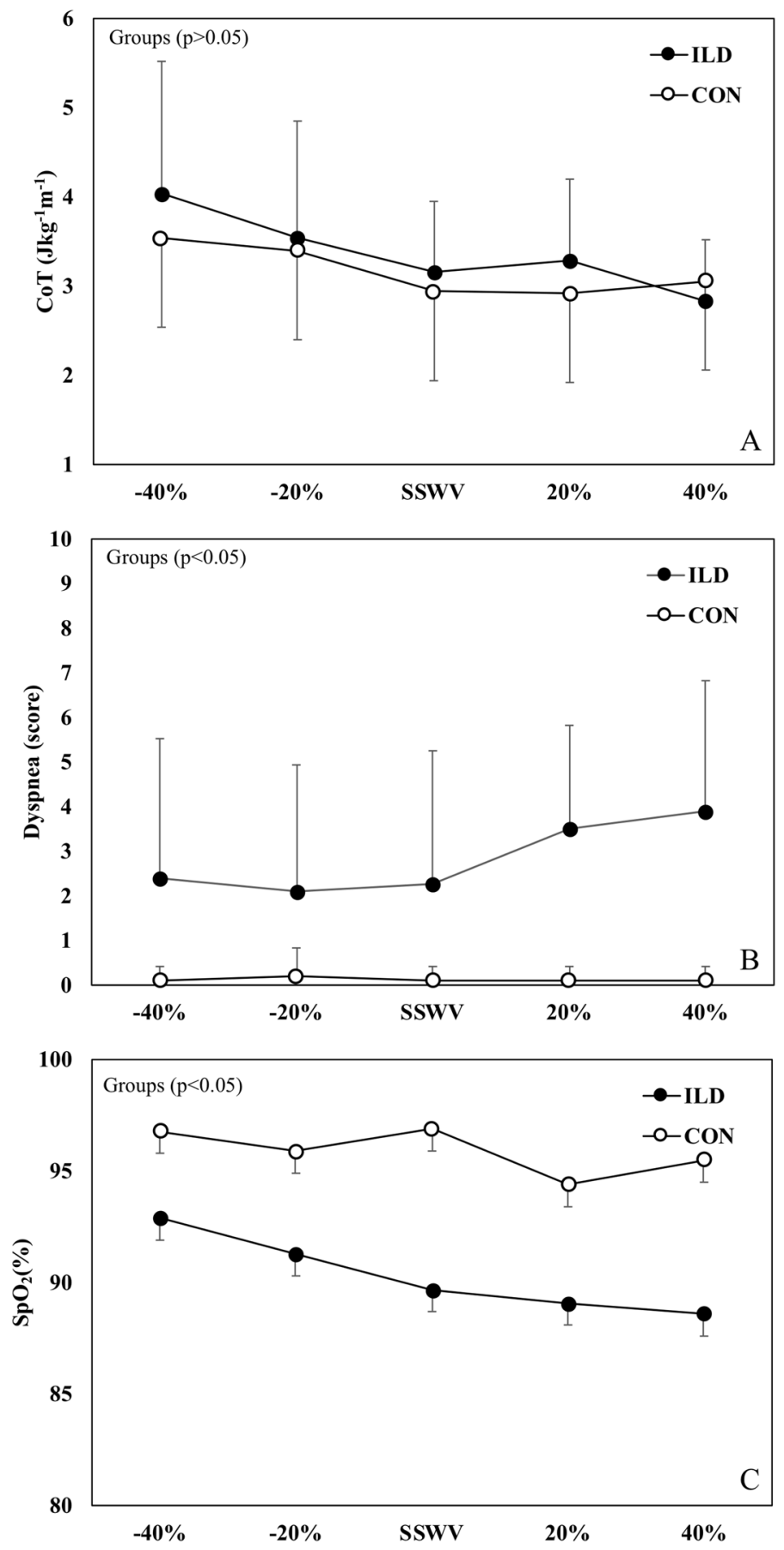

Figure 2. Cost of Transport (CoT; panel A), Dyspnea (panel B) and Oxyhemoglobin saturation by pulse oximetry (panel C) as a function of self-selected walking speed (SSWS), and two relative speeds below and two above SSWS in patients with interstitial lung disease (ILD, filled circles) and controls (CON, empty circles).

of speed $\times$ group, $p=0.337$ ). The average values ranged from 45.3 at $-40 \%$ speed to 40.7 at $+40 \%$ speed for the ILD group, and from 36.1 at $-40 \%$ speed to 30.6 at $+40 \%$ speed for the control group.

The CoV for contact time, swing time, and stride length were significantly higher in patients than in controls (Fig. 3), except for stride frequency $(p=0.058)$. CoVs with lower frequency and stride length were observed at higher speeds in the entire sample without interaction group vs. speed.

\section{Discussion}

The present study showed that patients with ILD adopted a lower SSWS than their OWS, in contrast to what was observed for controls. The perception of dyspnea was increased in ILD patients, especially at higher speeds, suggesting that higher breathlessness may discourage the adoption of higher (more energy-efficient) speeds. In 
Contact Time

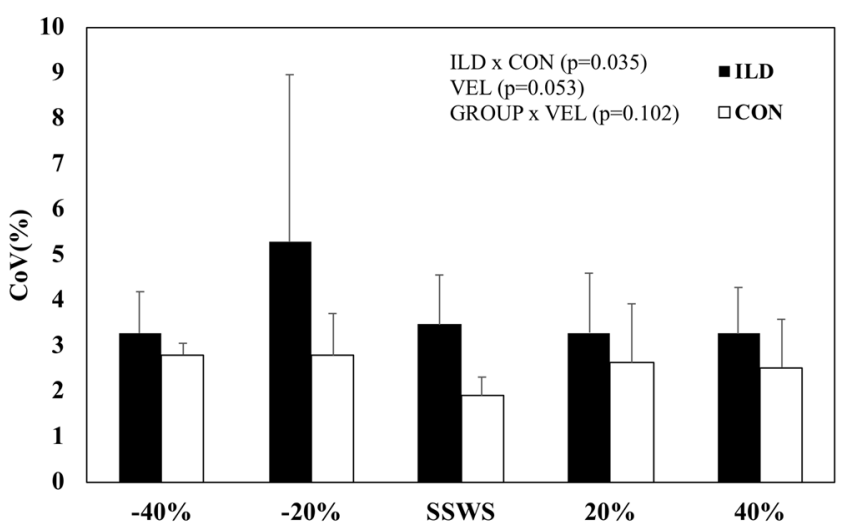

Stride Frequency

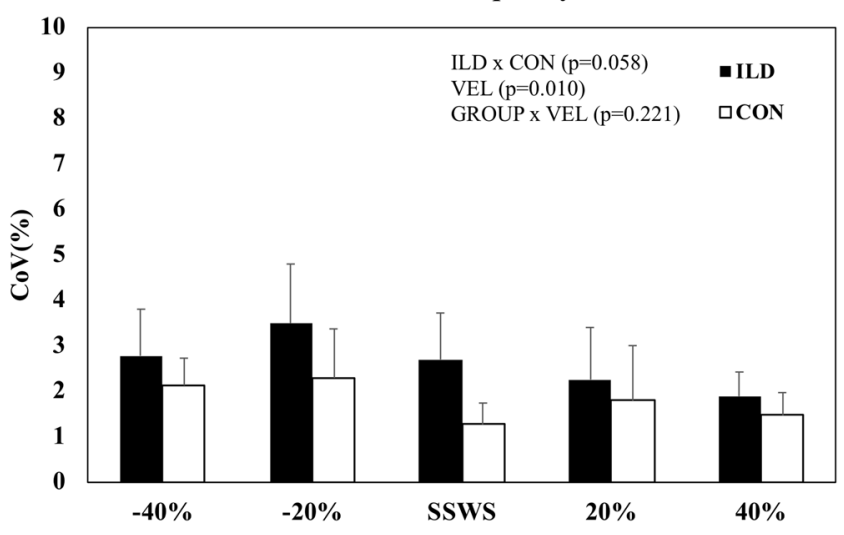

Swing Time

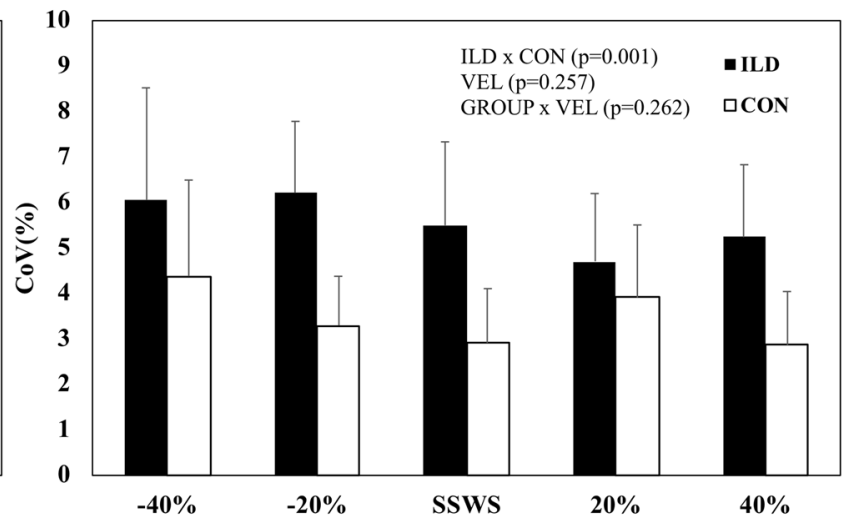

Figure 3. The coefficient of variation $(\mathrm{CoV})$ of contact time, swing time, stride frequency and stride length as a function of self-selected walking speed (SSWS), and two relative speeds below and two above SSWS in patients with interstitial lung disease (ILD, filled columns) and controls (empty columns). The main [group (ILD $\times$ CON) and velocity $(\mathrm{VEL})]$ and interaction $(\mathrm{GROUP} \times \mathrm{VEL})$ effects are denoted.

contrast, despite worse findings in ILD patients than in controls, parameters such as walking stability and dyspnea did not seem to sufficiently change at different speeds $(p>0.05)$ to a point believed to influence speed selection.

The CoT was not significantly different between the groups and among the speeds. This finding is similar to the results of results of a recent study on patients with COPD ${ }^{10}$. The increased cost of breathing observed in respiratory diseases seems to not be enough to impact the overall metabolic cost of walking ${ }^{35}$. These results support previous findings showing that the walking economy behavior in ILD resembles that observed in obstructive pulmonary disorders ${ }^{10}$. The SSWS of young and healthy people is around $4 \mathrm{~km} \mathrm{~h}^{-1}$ and is believed to be chosen because of the minimum CoT due to optimized pendulum mechanism ${ }^{13}$. Similar findings were observed in the elderly but the minimum CoT values were significantly higher compared to those in the younger individuals ${ }^{16}$. The presence of ILD altered this pattern. The patients' SSWSs were lower than those of the controls and different from their own OWS, which occurred at a higher speed. This means that the patients did not self-select their usual speed with the lowest CoT, as seen in the healthy individuals. The CoT U shape for ILD patients was uncertain because it was not feasible for them to walk at higher speeds $\left(>5 \mathrm{~km} \mathrm{~h}^{-1}\right)$. It is likely that the trend for an interaction effect group $\times$ speed effect $(p=0.051)$ could become significant with extra walking speeds.

Interestingly, based on locomotor rehabilitation index analyses, patients choose to walk at speeds around $50 \%$ of the biomechanical optimal speed. Moreover, recent findings showed an improved OWS in older people after Nordic walking training ${ }^{36}$. We speculate that interventions that enhance the speeds that cause moderate or higher dyspnea levels (e.g., Borg scores $\geq 3$ ) would increase the SSWS in individuals with ILD, positively affecting their daily activities.

To our knowledge, this is the first study to investigate dynamic walking stability in patients with ILD. The CoV results of the spatiotemporal variables of walking revealed lower dynamic stability in patients with ILD than in controls. According to Beauchet et al. ${ }^{37}$, because walking is a repetitive movement, its variability does not usually exceed $3 \%$. However, the $\mathrm{CoV}$ of the contact time and swing time resulted in mean values above $3 \%$ for ILD patients (potentially explained by deteriorated pendulum-like mechanism) ${ }^{32}$. This indicates a higher risk of falls, as observed in patients with COPD ${ }^{38-40}$. Our findings also revealed that walking below or above the SSWS can affect dynamic stability. Indeed, the $\mathrm{CoV}$ of stride frequency and length were higher at lower speeds in both the groups. The higher walking variability at low speeds is in line with previous findings of other cardiorespiratory 
disorders ${ }^{40}$ and stroke patients $\mathrm{s}^{34}$. It is also worth noting that the stride frequency variability values at SSWS (3\%) were higher than those reported by Sanseverino et al. $(1.7 \%)$ in COPD $^{10}$.

The causes of exertional dyspnea in chronic lung diseases are multifactorial. In particular, dyspnea occurs during exercise under conditions of an abnormal dynamic mechanical/muscular response of the respiratory system and increased central respiratory neural drive ${ }^{41}$. Although the ventilatory and perceptual responses to exercise stress are broadly similar between obstructive and restrictive lung diseases, our findings showed that ILD patients have an additional impairment in the peripheral muscular function (represented here as the dynamic stability) in comparison to obstructive pulmonary disorders ${ }^{10}$. The underlying causes of such differences are uncertain, but could be partly related to the lower cardiorespiratory fitness in ILD patients in the present study $\left(\dot{\mathrm{V}} \mathrm{O}_{2 \text { peak }}=13 \mathrm{~mL} \mathrm{~kg} \mathrm{~min}^{-1}\right)$ than in Sanseverino et al. ${ }^{10}\left(\dot{\mathrm{VO}}_{2 \text { peak }}=19 \mathrm{~mL} \mathrm{~kg}^{-1} \mathrm{~min}^{-1}\right)$.

Collectively, we speculate that the reduced SSWS is adopted to achieve the combination of the lowest dyspnea sensation (Fig. 2, panel B) despite higher overall gait instability (Fig. 3). Although higher speed would result in a higher economy, patients seem to choose a SSWS lower than the OWS based on lower dyspnea sensation. However, dyspnea was not significantly higher $(p>0.05)$ at higher speeds, probably because of the small sample size. Therefore, these results should be interpreted with caution.

This information may be helpful for physical activity improvement counseling. It may be worth advising patients to perform daily walking activities slightly faster than their SSWS, adopting frequent short pauses before tiring. Despite higher breathing discomfort, walking faster and, therefore, more economically, increases energy conservation and daily physical activity. This strategy needs to be confirmed in future studies using an appropriate randomized controlled trial.

Despite similar CoT, patients with ILD and healthy subjects have different walking strategies. Gait instability and dyspnea perception were significantly higher in patients with ILD. In this context of impaired walking stability, patients seem to adopt a lower SSWS compared to controls and their own OWS, resulting in lower dyspnea perception. From the physiomechanical point-of-view, impairments in gait stability and dyspnea perception are manifestations of disorders in the transmission and muscle efficiencies, respectively ${ }^{42}$. Therefore, ILD patients probably have an overall efficiency lower than controls despite the metabolic economy remaining similar.

\section{Limitations}

The present study had some limitations: the sample size was relatively small, mainly because the experimental design included treadmill tests, and the protocols (integrating biomechanical and physiological procedures) were rather long and wearing. Consequently, some patients could not be included. Walking at higher speeds $\left(>5 \mathrm{~km} \mathrm{~h}^{-1}\right)$ might have provided a clearer result for the CoT U shape but was not feasible for the ILD patients. In addition, future studies are warranted that use other measures such as the Lyapunov coefficient ${ }^{43}$ and margins of stability ${ }^{44}$ which should provide a deeper understanding of walking dynamical stability. Another limitation was the possible bioenergetic and biomechanical changes that occur during treadmill walking compared to overground walking ${ }^{45,46}$. However, despite these limitations, this study provides a valuable understanding of the SSWS adopted by patients, which could be considered in physical activity programs.

Ethics statement. This study was carried out in accordance with the recommendations of HCPA Committee with written informed consent from all subjects. All subjects gave written informed consent in accordance with the Declaration of Helsinki. The study protocol was approved by the ethics committee of Hospital de Clínicas de Porto Alegre.

Received: 20 August 2020; Accepted: 24 May 2021

Published online: 14 June 2021

\section{References}

1. Wells, A. U. \& Hirani, N. Interstitial lung disease guideline: The British Thoracic Society in collaboration with the Thoracic Society of Australia and New Zealand and the Irish Thoracic Society. Thorax 63(Supplement 5), 1-58 (2008).

2. Travis, W. D. et al. An Official American Thoracic Society/European Respiratory Society Statement: Update of the international multidisciplinary classification of the idiopathic interstitial pneumonias. Am. J. Respir. Crit. Care Med. 188, 733-748 (2013).

3. Morell, F. et al. Chronic hypersensitivity pneumonitis in patients diagnosed with idiopathic pulmonary fibrosis: A prospective case-cohort study. Lancet Respir. Med. 1, 685-694 (2013).

4. Holland, A. E. Exercise limitation in interstitial lung disease-mechanisms, significance and therapeutic options. Chron. Respir. Dis. 7, 101-111 (2010).

5. O'Donnell, D. E., Elbehairy, A. F., Berton, D. C., Domnik, N. J. \& Neder, J. A. Advances in the evaluation of respiratory pathophysiology during exercise in chronic lung diseases. Front. Physiol. 8, 82 (2017).

6. O’Donnell, D. E., Neder, J. A., Harle, I. \& Moran-Mendoza, O. Chronic breathlessness in patients with idiopathic pulmonary fibrosis: A major challenge for caregivers. Expert. Rev. Respir. Med. 10, 1295-1303 (2016).

7. Nolan, C. M. et al. Validity, responsiveness and minimum clinically important difference of the incremental shuttle walk in idiopathic pulmonary fibrosis: A prospective study. Thorax 73, 680-682 (2017).

8. Nolan, C. M. et al. Phenotypic characteristics associated with slow gait speed in idiopathic pulmonary fibrosis. Respirology 23, 498-506 (2018).

9. Hirabayashi, R. et al. The validity and reliability of four-meter gait speed test for stable interstitial lung disease patients: The prospective study. J. Thorac. Dis. 12, 1296-1304 (2020).

10. Sanseverino, M. A. et al. Limiting factors in walking performance of subjects With COPD. Respir. Care. 63, 301-310 (2018).

11. Margaria, R. Sulla fisiologia e specialmente sul consumo energético della marcia e della corsa a varia velocita' ed inclinazione del terreno. Att. Acc. Naz. Lincei. 7, 299-368 (1938).

12. di Prampero, P. E. The energy cost of human locomotion on land and in water. Int. J. Sports Med. 7, 55-72 (1986). 
13. Saibene, F. \& Minetti, A. E. Biomechanical and physiological aspects of legged locomotion in humans. Eur. J. Appl. Physiol. 88, 297-316 (2003).

14. Gast, K., Kram, R. \& Riemer, R. Preferred walking speed on rough terrain: Is it all about energetics?. J. Exp. Biol. 222, jeb185447 (2019).

15. Hunter, L. C., Hendrix, E. C. \& Dean, J. C. The cost of walking downhill: Is the preferredgait energetically optimal ?. J. Biomech. 43, 1910-1915 (2010).

16. Mian, O. S., Thom, J. M., Ardigò, L. P., Narici, M. V. \& Minetti, A. E. Metabolic cost, mechanical work, and efficiency during walking in young and older men. Acta Physiol. 186, 127-139 (2006).

17. Clark, B. C. \& Manini, T. M. Functional consequences of sarcopenia and dynapenia in the elderly. Curr. Opin. Clin. Nutr. Metab. Care 13, 271-276 (2010).

18. Kang, H. G. \& Dingwell, J. B. Effects of walking speed, strength and range of motion on gait stability in healthy older adults. J Biomech. 41, 2899-2905 (2008).

19. Watanabe, F. et al. Quadriceps weakness contributes to exercise capacity in nonspecific interstitial pneumonia. Respir. Med. 107, 622-628 (2013).

20. Panagiotou, M., Polychronopoulos, V. \& Strange, C. Respiratory and lower limb muscle function in interstitial lung disease. Chron. Respir. Dis. 13, 162-172 (2016)

21. Merola, P. K. et al. Relationship of pectoralis muscle area and skeletal muscle strength with exercise tolerance and dyspnea in interstitial lung disease. Sarcoidosis Vasc. Diffuse Lung Dis. 34, 200-208 (2017).

22. Nishiyama, O. et al. Quadriceps weakness is related to exercise capacity in idiopathic pulmonary fibrosis. Chest 127, 2028-2033 (2005).

23. Nolan, C. M. et al. Gait speed and prognosis in patients with idiopathic pulmonary fibrosis: A prospective cohort study. Eur. Respir. J. 53, $1801186(2019)$

24. Figueiredo, P., Ribeiro, P. A. B., Bona, R. L., Peyré-Tartaruga, L. A. \& Ribeiro, J. P. Ventilatory determinants of self-selected walking speed in chronic heart failure. Med. Sci. Sports Exerc. 45, 415-419 (2013).

25. Scano, G., Innocenti-Bruni, G. \& Stendardi, L. Do obstructive and restrictive lung diseases share common underlying mechanisms of breathlessness?. Resp. Med. 104, 925-933 (2010).

26. Faisal, A. et al. Common mechanisms of dyspnea in chronic interstitial and obstructive lung disorders. Am. J. Resp. Crit. Care Med. 193, 299-309 (2016).

27. Buchfuhrer, M. J. et al. Optimizing the exercise protocol for cardiopulmonary assessment. J. Appl. Physiol. 55, 1558-1564 (1983).

28. Borg, G. A. Psychophysical bases of perceived exertion. Med. Sci. Sports Exerc. 14, 377-381 (1982).

29. Guazzi, M. et al. 2016 Focused update: Clinical recommendations for cardiopulmonary exercise testing data assessment in specific patient populations. Eur. Heart J. 39, 1144-1161 (2018).

30. Marques A, et al. Reliability, Agreement and minimal detectable change of the timed up \& go and the 10-meter walk tests in older patients with COPD. COPD J. Chron. Obstr. Pulm. Dis. 13, 1-9 (2016).

31. Gomeñuka, N. A. et al. Nordic walking training in elderly, a randomized clinical trial. Part II: Biomechanical and metabolic adaptations. Sports Med. Open 6, 1-19 (2020).

32. Gomeñuka, N. A., Bona, R. L., da Rosa, R. G. \& Peyré-Tartaruga, L. A. Adaptations to changing speed, load, and gradient in human walking: Cost of transport, optimal speed, and pendulum. Scand. J. Med. Sci. Sports 24, 165-173 (2014).

33. Peyré-Tartaruga, L. A. \& Monteiro, E. P. A new integrative approach to evaluate pathological gait: Locomotor rehabilitation index. Clin. Transl. Degener. Dis. 1, 86-90 (2016).

34. Oliveira, H. B., Da Rosa, R. G., Gomeñuka, N. A. \& Peyré-Tartaruga, L. A. Estabilidade dinâmica da caminhada de indivíduos hemiparéticos: A influência da velocidade. Rev. Educ. Fis. 24, 559-565 (2013).

35. Sacco, O. L. et al. Tidal expiratory flow limitation induces expiratory looping of the alveolar pressure-flow relation in COPD patients. J. Appl. Physiol. 129, 75-83 (2020).

36. Gomeñuka, N. A. et al. Nordic walking training in elderly, a randomized clinical trial. Part II: Biomechanical and metabolic adaptations. Sports Med. Open 6, 1-19 (2020).

37. Beauchet, O. et al. Gait variability among healthy adults: Low and high stride-to-stride variability are both a reflection of gait stability. Gerontology 55, 702-706 (2009).

38. Roig, M., Eng, J. J., Road, J. D. \& Reid, W. D. Falls in patients with chronic obstructive pulmonary disease: A call for further research. Respir Med. 103, 1257-1269 (2009).

39. Smith, M. D., Chang, A. T., Seale, H. E., Walsh, J. R. \& Hodges, P. W. Balance is impaired in people with chronic obstructive pulmonary disease. Gait Posture 31, 456-460 (2010).

40. Yentes, J. M., Rennard, S. I., Schmid, K. K., Blanke, D. \& Stergiou, N. Patients with chronic obstructive pulmonary disease walk with altered step time and step width variability as compared with healthy control subjects. Ann. Am. Thorac. Soc. 14, 858-866 (2017).

41. Donnell, D. E. O., Ora, J., Webb, K. A., Laveneziana, P. \& Jensen, D. Respiratory physiology \& neurobiology mechanisms of activityrelated dyspnea in pulmonary diseases. Respir. Physiol. Neurobiol. 167, 116-132 (2009).

42. Peyré-Tartaruga, L. A. \& Coertjens, M. Locomotion as a powerful model to study integrative physiology: Efficiency, economy, and power relationship. Front. Physiol. 9, 1789 (2018).

43. Hof, A. L., Gazendam, M. G. \& Sinke, W. E. The condition for dynamic stability. J. Biomech. 38, 1-8 (2005).

44. Russo, L. et al. Shoes and insoles: The influence on motor tasks related to walking gait variability and stability. Int. J. Environ. Res. Public Health. 17, 5469 (2020).

45. Malatesta, D., Canepa, M., Fernandez, A. M. The effect of treadmill and overground walking on preferred walking speed and gait kinematics in healthy, physically active older adults. Eur. J. Appl. Physiol. 117, 1833-1843 (2017).

46. Zanardi, A. P. et al. Gait parameters of Parkinson's disease compared with healthy controls: A systematic review and meta-analysis. Sci. Rep. 11, 1-3 (2021).

\section{Acknowledgements}

We are grateful to Caroline Uber Ghisi, MSc for her medical assistance during the data collection and to Locomotion Group of UFRGS for discussions and comments. This study was supported by a grant from the Hospital de Clínicas de Porto Alegre Fund for the Incentive of Research, Porto Alegre, Brazil, G. F. and F. B. Q. received scholarships from Brazilian Coordination for the Development of Superior Education Personnel (CAPES), Brasília, Brazil. L.A. P.-T. is a fellow of CNPq Research Productivity, level 2, Brazil.

\section{Author contributions}

G.F., F.B.Q. and L.A.P.T. conceived of the study and designed the experiments. L.A.P.T. and D.C.B. obtained the funding. G.F., F.B.Q, H.B.O. and L.A.P.T. carried out the analysis, interpreted the statistical results, and drafted the 
manuscript. G.F., F.B.Q, M.C. and P.S., collected the data. G.F., P.S., M.C., D.C.B., M.G. and L.A.P.T. contributed to the manuscript to the formal analysis, writing, read, and approved the final manuscript.

\section{Competing interests}

The authors declare no competing interests.

\section{Additional information}

Supplementary Information The online version contains supplementary material available at https://doi.org/ 10.1038/s41598-021-91734-X.

Correspondence and requests for materials should be addressed to L.A.P.-T.

Reprints and permissions information is available at www.nature.com/reprints.

Publisher's note Springer Nature remains neutral with regard to jurisdictional claims in published maps and institutional affiliations.

(c) (i) Open Access This article is licensed under a Creative Commons Attribution 4.0 International License, which permits use, sharing, adaptation, distribution and reproduction in any medium or format, as long as you give appropriate credit to the original author(s) and the source, provide a link to the Creative Commons licence, and indicate if changes were made. The images or other third party material in this article are included in the article's Creative Commons licence, unless indicated otherwise in a credit line to the material. If material is not included in the article's Creative Commons licence and your intended use is not permitted by statutory regulation or exceeds the permitted use, you will need to obtain permission directly from the copyright holder. To view a copy of this licence, visit http://creativecommons.org/licenses/by/4.0/.

(C) The Author(s) 2021 